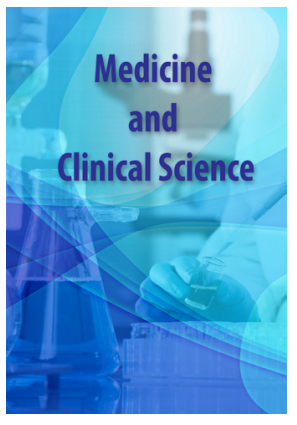

Correspondence

Catherine Michaux

Laboratoire de Chimie Physique des Biomolécules, Unité de Chimie Physique

Théorique et Structurale (UCPTS), University of Namur, Rue de Bruxelles, 61, 5000 Namur, Belgium

Tel.: +32 81724557

E-mail: catherine.michaux@unamur.be

- Received Date: 25 Sep2019;

- Accepted Date: 19 Oct 2019;

- Publication Date: 30 Oct 2019.

\section{Copyright}

(c) 2019 Science Excel. This is an openaccess article distributed under the term of the Creative Commons Attribution 4.0 International license.

\title{
Unravelling the refolding of membrane proteins, one of the most challenging therapeutic targets
}

\author{
Catherine Michaux ${ }^{1,2,3^{*}}$ and Eric A Perpète ${ }^{1,2,4}$ \\ 'Laboratoire de Chimie Physique des Biomolécules, Unité de Chimie Physique Théorique et Structurale (UCPTS), University of \\ Namur, Rue de Bruxelles, 61, 5000 Namur, Belgium \\ ${ }^{2}$ Namur Institute of Structured Matter, University of Namur, Rue de Bruxelles, 61, 5000 Namur, Belgium \\ ${ }^{3}$ Namur Research Institute for Life Sciences, University of Namur, Rue de Bruxelles, 61, 5000 Namur, Belgium \\ ${ }^{4}$ Institute of Life-Earth-Environment, University of Namur, Rue de Bruxelles, 61, 5000 Namur, Belgium
}

\section{Introduction}

Being involved in many essential cellular processes, Membrane Proteins (MPs) constitute one of the most important therapeutic targets, such as $\mathrm{G}$ protein-coupled receptors (GPCR), small molecule transporter or ion channels [1-3]. A detailed knowledge of their structure and activity is essential to design new therapeutic or biomimetic agents and therefore plays a significant role in the drug discovery process.

The major difficulties frequently encountered during MPs in vitro study are mainly due to their partially hydrophobic surfaces, flexibility and lack of stability. As a consequence, less than $1 \%$ of all solved protein crystal structures are MPs [4], witnessing how challenging is their expression, solubilisation, purification and crystallisation. Protein overexpression in E. coli is a very common method as it is quite inexpensive, easy to use, and can provide results in a reasonable lapse of time. However, the production of MPs from bacterial membrane is often toxic, and generally expression yields tend to be limited.

A well-established alternative is based on the formation of inactive aggregates called inclusion bodies (IBs) in the cell cytosol. Subsequently, a refolding step from IB is needed but it often turns to be a difficult task [5]. Whilst the refolding of soluble globular proteins from IB has become routine, much fewer examples of MPs in vitro refolding have indeed been reported.

Conventional protocols include solubilizing IB with high concentrations of denaturant, followed by the lowering of the denaturant concentration, and the addition of detergents that simulate the membrane environment. Unfortunately, this approach frequently fails due to the disposition of proteins to aggregate upon the removal of denaturant, and one has to fall back on a time-consuming and inefficient trial-and-error strategy. In order to circumvent these shortcomings, the development of new efficient refolding techniques is urgently awaited. That is, the real challenge is to develop universal methods for MPs.

The so-called artificial chaperone-assisted refolding procedure based on the stripping-off effect of cyclodextrins has already been successfully used to refold several types of globular proteins, but up to now only few publications refer to the adaptation of this protocol to MPs [6]. For instance, the amphipathic polymer Apol A8-35 is able to refold both $\alpha$-helical and $\beta$-barrel MPs [7] though solubility issues may arise in particular conditions. Other non-conventional approaches were developed to stabilize MPs: nanodiscs, fluorinated surfactants, lipopeptides, but none of them has proved its abilities to refold several types of MPs [8].

In that context, we have successfully demonstrated the reliability of a genuine protein refolding procedure based on the synergistic association of a detergent and a co-solvent.

\section{Results}

The powerful denaturant Sodium Dodecyle Sulfate (SDS), and the 2-Methyl-2,4-PentaneDiol (MPD) diol-type co-solvent are both known to affect the structure and solubility of proteins. More astonishing is that they show totally unexpected properties when combined in appropriate proportions. An optimal concentration of MPD, i.e. $1-1.5 \mathrm{M}$, seems to be essential for the refolding, whatever the protein, in conjunction with $\mathrm{mM}$ concentrations of SDS

Remarkably, our method has successfully been applied to several peptides and proteins featuring diverse structures and properties [9-14]. Indeed, several monomeric $\beta$-barrel MPs with a variable number of transmembrane (TM) strands have been refolded: PagP (8TM), a VDAC (19TM), FhuA (22TM). Oligomeric MPs have also been recovered: the heptameric hemolysin (14TM) and the trimeric Omp2a (16TM). Aside, our seminal technique was recently used for diverse applications, taking advantage of its refolding, solubilizing and stabilizing properties [10,15-17]. Moreover, crystal structures of both the globular hen-egg lysozyme [18] and the membrane protein PagP [19] have clearly established the preservation of the native state in a SDS-MPD mixture. They also show that MPD lowers or annihilates the interactions between the denaturant and the protein.

Unlike the other refolding methods, the originality of our process is that MPD is used to modify the 
denaturant properties without having to reduce its concentration. MPD is able to transform the harsh denaturing SDS character into a gentle non-denaturing detergent that softly interacts with the protein. Furthermore, this method has been demonstrated to be compatible with various characterization techniques, such as Circular Dichroism, Dynamic Light Scattering, fluorescence, crystallography and transmission electron microscopy. On the top of that, our protocol is particularly inexpensive with respect to other available techniques.

Combining spectroscopic and computational approaches, we have refined our understanding of the molecular interactions underlying the SDS-MPD association [20-22]. By shielding the negative charge on SDS headgroups and protecting the hydrophobic tail of the detergent from water, MPD molecules can separate SDS molecules from each other, leading to the SDS micelle dismantlement. In a first stage, the aggregation of proteins is prevented by the addition of detergent molecules that shield the hydrophobic regions of the denatured protein. Afterwards, the co-solvent alters the surfactant denaturing properties by stripping it away from the protein-detergent complexes, and allowing the subsequent refolding. The performance and specific properties of the co-solvent MPD are hypothesized to rely on its specifically balanced lipophilic-hydrophilic character.

\section{Perspectives}

The efficient and inexpensive SDS-MPD method has already given promising results on the refolding of $\beta$-barrel MPs. Having already demonstrated that MPD is able to protect the a-helical bacteriorhodopsin [9], we now intend to refold such type of MPs, like GPCRs, that will consequently be available for analysis and pave the way towards the design of new drugs.

\section{Acknowledgments}

C.M. and E.A.P. thank the Belgian National Fund for Scientific Research for their research associate and senior research associate positions, respectively.

\section{References}

1. Overington JP, Al-Lazikani B, Hopkins AL. How many drug targets are there? Nat Rev Drug Discov 2006, 5:993-96.

2. Yin $\mathrm{H}$ and Flynn AD. Drugging Membrane Protein Interactions. Annu Rev Biomed Eng 2016, 18(1):51-76.

3. Gong J, Chen Y, Pu F, et al. Understanding Membrane Protein Drug Targets in Computational Perspective. Curr Drug Targets 2019, 20(5):551-64.

4. White SH. 2015. Membrane Proteins of Known 3D Structure. http:// blanco.biomol.uci.edu/mpstruc/

5. Hattab G, et al. Escherichia coli as host for membrane protein structure determination: a global analysis. Sci Rep 2015, 5:12097.
6. Michalke K, Warschawski ED, Moncoq K, et al. Escherichia coli as host for membrane protein structure determination: a global analysis. Anal Biochem 2010, 401(1):74-80.

7. Le Bon C, Marconnet A, Masscheleyn S, et al. Folding and stabilizing membrane proteins in amphipol A8-35. Methods 2018, 147:95-105

8. Popot JL. Amphipols, nanodiscs, and fluorinated surfactants: three nonconventional approaches to studying membrane proteins in aqueous solutions. Annu Rev Biochem 2010, 79:737-75.

9. Michaux C, Pomroy NC, Privé GG. Refolding SDS-denatured proteins by the addition of amphipathic cosolvents. J Mol Biol 2008, 375(5):1477-88.

10. Kinzel J, Sauer DF, Bocola M, et al. 2-Methyl-2,4-pentanediol (MPD) boosts as detergent-substitute the performance of $\beta$-barrel hybrid catalyst for phenylacetylene polymerization. Beilstein J Org Chem 2017, 13:1498-506.

11. Tanaka Y, Hirano N, Kaneko J, et al. 2-Methyl-2,4-pentanediol induces spontaneous assembly of staphylococcal a-hemolysin into heptameric pore structure. Protein Sci 2011, 20(2):448-56.

12. Roussel G, Perpète EA, Matagne A, et al. Towards a universal method for protein refolding: the trimeric beta barrel membrane Omp2a as a test case. Biotechnol Bioeng 2013, 110(2):417-23.

13. Roussel G, Tinti E, Perpète E, et al. Refolding of SDS-denatured proteins using amphipathic cosolvents and osmolytes. Curr Protoc Protein Sci 2013; 72(1): 28.5.1-28.5.9.

14. Michaux C, Roussel G, Lopes-Rodrigues $M$, et al. Unravelling the mechanisms of a protein refolding process based on the association of detergents and co-solvents. J Pept Sci 2016, 22(7):485-91.

15. Garakani TM, Richter MJ, Böker A. Controlling the bio-inspired synthesis of silica. J Colloid Interface Sci 2017, 488:322-34.

16. Charan $\mathrm{H}$, Kinzel J, Glebe U, et al. Grafting PNIPAAm from $\beta$-barrel shaped transmembrane nanopores. Biomaterials 2016, 107:115-23.

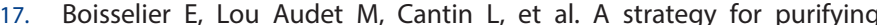
glutathio S-transferase in the presence of sodium dodecyl sulfate. Biotechniques 2011, 51(3):193-4.

18. Michaux C, Pouyez J, Wouters J, et al. Protecting role of cosolvents in protein denaturation by SDS: a structural study. BMC Struct Biol 2008, 8:29.

19. Cuesta-Seijo JA, Neale C, Khan MA, et al. PagP crystallized from SDS/ cosolvent reveals the route for phospholipid access to the hydrocarbon ruler. Structure 2010, 18(9):1210-9.

20. Roussel G, Caudano Y, Matagne A, et al. Peptide-surfactant interactions: A combined spectroscopic and molecular dynamics simulation approach. Spectrochim Acta A Mol Biomol Spectrosc 2018, 190:464-70.

21. Roussel G, Michaux C, Perpète EA. Multiscale molecular dynamics simulations of sodium dodecyl sulfate micelles: from coarse-grained to all-atom resolution. J Mol Model 2014, 20(10):2469.

22. Roussel G, Rouse SL, Sansom MS, et al. The role of 2-methyl-2, 4-pentanediol in sodium dodecyl sulfate micelle dissociation unveiled by dynamic light scattering and molecular dynamics simulations. Colloids Surf B Biointerfaces 2014, 114:357-62. 